\title{
Piroxicam- Induced Hepatotoxicity
}

\author{
Omar Rashid Sadeq* \\ Assistant Professor, Faculty of Dentistry, Arab American University Jenin, (AAUJ) Palestine
}

Received: January 28, 2018; Published: February 12, 2018

*Corresponding author: Omar Rashid Sadeq, Assistant Professor, Faculty of Dentistry, Arab American University Jenin, (AAUJ) Palestine, Email: goldman2007@bigmir.net

\begin{abstract}
Piroxiam is an oxicam derivative medication belonging to non steroidal anti-inflammatory drugs (NSAIDs) group, used to treat moderate to severe inflammatory diseases such as rheumatoid arthritis, osteoarthritis, ankylosing spondylitis (Bechterew's disease), tendinitis, bursitis, and for pain that is not related to musculoskeletal system e.g. primary dysmenorrhea and postoperative pain. It reduces pain, joint swelling, morning stiffness, and improves the functionality of the joints during chronic polyarthritis. Piroxicam has been compared to other antiinflammatory agents (e.g. diclofenac, indomethacin, and naproxen) in numerous controlled studies and proved to be equal and sometimes even more efficacious. Piroxicam is unique among NSAIDs in that it is used once daily, and its efficacy is equal to most important clinically employed NSADs (ibuprofen, naproxen and diclofenac) especially in amelioration of postoperative pain, but it is more prone to causing gastrointestinal disturbances and serious skin reactions (Steven-Johnsons syndrome, toxic epidermal necrolysis). It should not be given to patients who have experienced peptic ulcer, asthma, urticaria or allergic-type reactions after taking aspirin or other NSAIDs, as well as patients on anticoagulant therapy, renal, hepatic, diabetic and cardiac patients.

Piroxicam is not recommended for use in lactation, children under 12 years of age and pregnant women since safety has not been established as with any NSAID, caution should be exercised in treating the elderly (65 years and older). Piroxicam is provided in much trade formulation namely feldene, Felcol and pirox, it is available in multiple dose variations 10-20 mg capsules P0 1-2 times/day (no more than 30-40 mg/day) and is taking with or after food, the dose schedule is individual and depends on pathologic condition. Clinically significant drug interactions with piroxicam include bleeding when used with either anticoagulants or other NSAIDs, decreased efficacy of antihypertensive (ACEIs and beta blockers but not Ca channel blockers), reduced the antinatriuretic effect of diuretics such furasemide and hypothiazide, acceleration of the steady state of digoxin, increased nephrotoxicity of methotrexate and cyclosporine and finally concomitant use of corticosteroids with Feldene may increase the risk of GI ulceration or bleeding. The aim of recent study was to estimate the hepatic risk associated with the use of piroxicam, about 32 patients with osteoarthritis (OA) were divided into 2 categories, and were treated by piroxicam, 10-20 mg/d orally in a period of 2 months, patients were investigated for 6 month. CBC, hepatic tests including (bilirubin, ALT and AST) were done before, during and after treatment, ultrasonography and liver biopsy was also provided only for certain patients of II category. The main findings of this research study is that two-month orally prescribed piroxicam, $10 \mathrm{mg} / \mathrm{d}$ produces no hepatotoxic effect in OA, but piroxicam, $20 \mathrm{mg} / \mathrm{d}$, produces a mixed hepatocellular-cholestatic reversible injury in about $75 \%$ of OA patients, predominantly in female gender.
\end{abstract}

Keywords: Piroxicam; NSAIDs; Osteoarthritis; Rheumatoid Arthritis; Dysmenorrhea; Polyarthritis; Steven-Johnsons syndrome; Furasemide Hypothiazide; Methotrexate; Digoxin; ultrasonography; cholestasis; Liver Biopsy

Abbreviations: DILI: Drug Induced Liver Injury; NSAIDs: Non-steroidal anti-inflammatory drugs, CIX: Cyclooxygease; ALT: Alanine Amino Transferase; CBC: Complete Blood Count; RF: Rheumatoid Factor; ALP: Alkaline Phosphatase

\section{Introduction}

The liver plays an astonishing array of vital functions in the maintenance, performance and regulating homeostasis of the body, its major functions are immunity, carbohydrate, protein and fat metabolism, exogenous (drug) and endogenous substances detoxification, secretion of bile and storage of vitamin. More than 900 drugs have been implicated in causing liver injury and it is the most common reason for a drug to be withdrawn from the market. Drug Induced Liver Injury (DILI) is progressively increased, general pathophysiologic mechanisms involved in DILI include [1-5], a) Direct injury of hepatocytes with their membrane rupture

b) Interruption of bile flow via blocking of transport proteins at the canalicular membrane

c) Apoptosis of hepatocytes

d) Immunologic when a drug act an immunogen, and can affect the P450 system

e) Bile duct injury, the most commonly DILI are antibiotics, 
NSAIDs, anesthetic agents, antihyperlipedmics, antirheumatic drugs, TNF inhibitors, antiepileptics, antpsychotic drugs acetyl cholinesterase inhibitors, tricyclic antidepressants and antihypertensive agents [6-10].

Non-steroidal anti-inflammatory drugs (NSAIDs) are consumed massively worldwide and, along with antimicrobial agents, are the most frequent causes of drug-induced liver injury. The pharmacology of NSADs is broad and diverse, their principle effects are analgesic, antipyretic and anti-inflammatory, these effects are highly advantages in the clinical settings, so many patients are afflicted by one or more of these symptoms. For this reason NSAIDs find use in conditions such as toothache, arthralgia, myalgia, headache, migraine, fever, to inflammatory arthritic diseases such as rheumatoid arthritis, osteoarthritis, gout, to postoperative pain.

More than 20 different NSAIDs are available commercially, and these agents are used worldwide, for their three above mentioned therapeutic effects in patients with multiple medical conditions. The prototype is aspirin, commonly prescribed NSAIDs are diclofenac $\mathrm{K}$ or $\mathrm{Na}$, ibuprofen, indomethacin, piroxicam, naproxen, meloxicam and etoricoxib, and they share a common mode of action which involves blocking cyclooxygease (COX) enzymes [1116]. Different NSAIDs inhibit COX isoenzymes COX-1, COX-2 to different extents and this differential mechanism of action explains their differing wanted therapeutic effects and unwanted adverse actions. Acetaminophen (paracetamol) is not considered as NSAIDs as it has negligible anti-inflammatory powers even at high doses, (compared to aspirin), its analgesic and antipyretic effects are due to central inhibition of COX-1.

COX-1 isoenzyme is a constrictively expressed, because it is involved in many physiological processes for instance GIT mucosa protection, platelets aggregation and potency of blood vessels. COX-2 in contrast to COX-1 is facultatively expressed mainly during inflammatory states, but this does not exclude its physiologic role in CNS, macula densa of renal tissues as well as ovaries and uterus. Most NSAIDs competitively inhibit both isoenzymes to some degree, though aspirin- as an exception irreversibly blocks its target. COX inhibition is vital, as its COX enzymes are responsible for generation of prostanoids-substances which include prostaglandins "PGs" (implicated in inflammation and anaphylaxis), prostocyclines (active in resolution phase of inflammation) and thromboxanes (mediators of vasoconstriction). Nonselective COX-1/COX-2 inhibitors e.g. aspirin, piroxicam and naproxen- target COX-1 and as a result gastric PGs levels are reduced and for this reason GIT symptoms are considerably more common, ranging from mild erosions to severe bleeding, about $15 \%$ of patients experience dyspepsia on NSAIDs, the use of misoprostol (PG analogue) with naproxen, diclofenac or aspirin protects GIT mucosa from ulcerogenic effects of NSAID [17-22].

Their antipyretic effect is due to inhibition of PGE-2 synthesis form the thermoregulatory center- the hypothalamus, but not hyperthermia in which the set point is not altered. Selective COX-2 inhibitors such as celecoxib, valdecoxib and meloxicam in low doses are superior to nonselective in that they have less GIT distress, without affecting the bleeding time and therefore preferred for patients suffering from GIT and bleeding disorders, on the other hand selective COX-2 inhibitors shouldn't be used in CNS and renal diseases.

They kill pain peripherally due to their ability to minimize sensitization of receptors to bradykinin and PGs, but not centrally as do opioids that interrupt pain transmission at the level of CNS, NSAIDs (non opioid narcotics) differ from opioids e.g. (morphine or fentanyl) in that they do not produce a ceiling effect, as well as NSAIDs don't affect the emotional aspect of pain and. COX inhibitors as monotherpay are ineffective in reducing visceral, spastic, ischemic, necrotic and neoplastic pains. Sometimes they are combined with opioids to reduce pain arising from nonintegumental structures, and finally addiction and tolerance are specific for opioids rather than NSADs [23-30].

Pharmacokinetic ally, NSAIDs are well absorbed from the gastrointestinal tract, with the exception of aspirin (and possibly diclofenac) which undergoes presystemic hydrolysis to form salicylic acid. Concomitant administration of NSAIDs with food or antacids may in some cases lead to delayed or even reduced absorption, they are highly bound to plasma proteins (mainly albumin), which limits their body distribution to the extracellular spaces, undergo hepatic transformations variously by CYP2C8, 2C9, 2C19 and/or glucuronidation. Half-lives of the NSAIDs vary but in general can be divided into "short-acting" (less than 6 hours, including ibuprofen, diclofenac and indomethacin) and "long-acting" (more than six hours, including naproxen, celecoxib, meloxicam and piroxicam).The elimination of these drugs depends largely on hepatic biotransformation; renal excretion of unchanged drugs is usually small (less than $5 \%$ of the dose). NSAIDs differ in potency, duration of action, side-effect profile, and potential for drug interactions, the selection of NSAID should be based on clinical experience, patient convenience (e.g. once or twice daily dosage schedule), side effects and cost. Despite the increasing number of NSAIDs available, there are few data comparing the old and new agents for efficacy and safety, and there are few guidelines governing choices of NSAIDs for particular patients [31-37].

Many studies show that aspirin, indomethacin, naproxen, fenoprofen, and ibuprofen were equally effective in the treatment of RA. Other studies for management of osteoarthritis reveal that indomethacin, naproxen, isoxicam (chemical analogue of piroxicam) and ketoprofen are equal in efficacy but the latter three had fewer side effects than indomethacin. Naproxen and aspirin are preferred for the treatment of muscle contraction headache, whereas indomethacin should be avoided, in contrast, indomethacin is the drug of choice for chronic paroxysmal hemicrania and hemicrania continua. Studies show that piroxicam (20mg /day) compared to other NSAIDs is more potent and less frequently employed daily, because of its long half life, notably piroxicam in RA is equal to ibuprofen (400 mg 3-4 times a day), but better than indomethacin (25 mg administered three times daily). In OA, piroxicam is slightly superior to naproxen (500 mg B.I.D). There is a high degree of "crosssensitivity" between aspirin and other NSAIDs in patients who 
have symptoms of rhinitis or asthma, the genesis is pharmacologic rather than immunologic, compared to urticaria (upon exposure to aspirin) in which mechanism is probably is immunologic (salicylate metabolite), that does not correlate with other NSAIDs [38-43].

There is no proved advantage to using more than one NSAID at a time unless a rapid onset of action is needed. If one drug does not prove efficacious after 1-3 weeks at the maximally tolerated dose, another agent should be substituted. For patients with gastric intolerance to one NSAID, alternative therapy from another class should be considered. If unsuccessful, therapy with choline salicylate, salsalate or enteric-coated aspirin may prove useful. When adverse effects of NSAIDs on platelets are of concern, sulindac or ibuprofen should be considered, with nonacetylated salicylates as alternatives. If renal function is compromised, avoid NSAIDs, especially fenoprofen if possible; sulindac is perhaps the least offensive agent, but close monitoring should be instituted. When central nervous system side effects such as headache occur, aspirin or naproxen may be used. In hypertension the pressor effect of NSAIDs could be minimized by prescribing sulindac and avoiding indomethacin. Paracetamol is still the only analgesic choice for asthmatic patients. So the choice of any member of NSAIDs should be done carefully, assuming to the above mentioned factors.

\section{Methodology}

About 32 primary knee joint OA patients aged from 30 to 60 years, were investigated for possibility of piroxiam-induced hepatitis in the period of 6 months, 16 of them are males, according to $\mathrm{OA}$ stage and symptoms piroxicam, $10 \mathrm{mg}-20 \mathrm{mg} / \mathrm{d}$ was given for 2 months, because its therapeutic effects become evident, only after the first 8-12 weeks of treatment, the patients were categorized into 2 categories: The first category includes 16 patients with the (mild II stage) of OA, 9 of them are women, medicated orally by piroxicam,10 mg/day. The second category contains 16 patients with the (III stage moderate) of OA, 10 of them are women; they were given piroxicam $20 \mathrm{mg} / \mathrm{d}$. per.os. Complete blood count (CBC), the rheumatoid factor, (RF), hepatic tests functions including bilirubin, alanine aminotransferase (ALT), and alkaline phosphatase (ALP) were done before during and upon completion of treatment in all patients. All patients were investigated for possibility of liver injury effect of piroxicam at the first 3-4 weeks following treatment. At the end of treatment, iron, ferttin, ultrasonography (US), serologic reactions and liver biopsy were indicated only to 11 jaundiced patients of the second category [44-47].

\section{Methods of Investigation \& Discussion}

The first category of patients experience minor knee pain after walking and running, joint stiffness and tenderness, they were medicated by proxicam, $10 \mathrm{mg} / \mathrm{d}$ for 2 months, the usual dose of piroxicam for $\mathrm{OA}$ arthritis is $20 \mathrm{mg} / \mathrm{d}$, but many studies show that giving piroxicam, $10 \mathrm{mg} / \mathrm{d}$ was successful in a small number of arthritic patients, the other purpose why we gave these patients Table 1: Hematologic and Hepatic Tests of Patients of I Group Medicated by Piroxicam. this dose was to determine if piroxicam is dose related toxicant or not. Patients of the second category clinically complain of more intense symptoms compared to the first one, they manifest acute knee joint pain with swelling, the pain is exacerbated by motion and relieved by rest, night pain is said to be present in almost patients especially patients above $55 \mathrm{y}$, ( 8 females, 3 males), joint pain is typically accompanied by morning stiffness and generally lasts less than an hour, they also experience a decreased range of motion and muscle spam.

On examination, patients of II category demonstrate localized tenderness along the joint, osteophytes is palpable around the affected knee joint, before treatment, by piroxicam, all patients of I and II groups were investigated for possibility of the presence of cardiovascular, endocrine, hepatic, skin and renal diseases, all of which were excluded, a detailed medical history including thorough questioning about medical factors, risk factors, use of prescription drugs, self-medication, and use of unconventional substances such as alternative and herbal medicine was provided with negative answers. RF was negative in both categories. Investigations show that treatment by piroxicam in both categories, equally and markedly decrease signs of acute inflammatory process of knee joint. Patients of I group after treatment demonstrate improvement of motility, ability to flex or extend their knees, as well as decreased tenderness and joint stiffness. Along a period of two month treatment, only 2 of 16 patients demonstrate mild dyspepsia, abdominal pain, reflecting the ulcerogenic effect of piroxicam on GIT mucosa, any change in the skin or eye pigmentations are not noted in all patients of this category $[48,49]$.

Piroxicam given to this group for 2 month at a dose $10 \mathrm{mg} / \mathrm{d}$ markedly decreases inflammatory reactions of $\mathrm{OA}$, that was reflected on $\mathrm{CBC}$, in which leukocytosis and ESR are normalized, indicating the efficacy of piroxicam, against OA, without any side effect on GIT mucosa, (except only in 12,5\% of patients dyspepsia appears, as mentioned above), because hepatic tests (bilirubin, and hepatic tests before, during and after treatment have no any significant deviation, therefore piroxicam, $10 \mathrm{mg} / \mathrm{d}$ for 2 month produces a strong effect against $\mathrm{OA}$ has no hepatotoxic effect (Table 1). Concerning the second category, piroxicam, $20 \mathrm{mg} / \mathrm{d}$ exerts a strong analgesic and anti-inflammatory effects in all patients, confirmed on $\mathrm{CBC}$, by decreasing WBCs count as well as normalization of ESR, most patients demonstrate that motion becomes more better than before treatment, swelling, morning stiffness, night pain and muscle spasm are significantly diminished. Only 4 patients of this group demonstrate nausea and vomiting, other complaints are not observed, their hematologic analysis and biochemistry show no any significant deviation, before, during and after treatment. About 12 patients of this category ( 9 females, 3 males ) at the end of eight week of course treatment complained abdominal tenderness, fatigue, nausea, vomiting, generalized pruritus, jaundice, dark urine and pale stool, At this period, piroxicam therapy was stopped.

\begin{tabular}{|c|c|c|c|}
\hline Hematologic \& Hepatic Tests $\boldsymbol{\otimes}$ & Normal Values & Before Treatment & After treatment \\
\hline WBCs & $4,1-10,9 \mathrm{~K} / \mathrm{uL}$ & $14-18$ & $5-10$ \\
\hline
\end{tabular}




\begin{tabular}{|c|c|c|c|}
\hline \multirow{2}{*}{ ESR } & M: $1-20 \mathrm{~mm} / \mathrm{hr}$ & M: $20-24$ & M: $1-16$ \\
\cline { 2 - 4 } & F: $1-30 \mathrm{~mm} / \mathrm{hr}$ & F: $22-39$ & F: $4-24$ \\
\hline ALT & $<40$ & 36 & 38 \\
\hline ALP & $<280$ & 259 & 270 \\
\hline Bilirubin $(\mathrm{mg} / \mathrm{dL})$ & $1.2 \mathrm{mg} / \mathrm{dL}$ & 0.8 & 1.1 \\
\hline
\end{tabular}

\section{N.B}

$\mathrm{K} / \mathrm{uL}=$ one $\mathrm{ul}$ is equal to $\mathrm{mm}$ (3) $\mathrm{K}$ means a thousand (1000 cells/ul).

凶: the symbol refers to mean average of laboratory values among 16 patients.

ALT: Alanine aminotransferase, ALP: Alkaline phosphatase, M: Male, F: Female.

The mean average of biochemical analysis was taken from the 12 jaundiced patients of the II category showed, increased total concentration of bilirubin, $(5.9 \mathrm{mg} / \mathrm{dl})$, increased serum of bilirubin accounts for conjugated form, ALT and ALP levels are also increased (694, $575 \mathrm{U} / \mathrm{L}$ respectively), with normal CBC. Ultrasonography (ultrasound) showed a normal liver, gallbladder and biliary tree. Liver biopsy reveals intrahepatic cholestasis with only mild inflammation and hepatocellular necrosis (Table 2).
Patients presented by acute symptoms of suspected hepatic injury at the end of the $8^{\text {th }}$ week of treatment by piroxicam, had no history about hepatic diseases, they didn't take any medication for the last six month, serologic reactions to hepatitis A, B, C, cytomegalovirus, Herpes Simplex, Epstein-Barr virus were negative, values of iron and ferrtin are within the reference range, so excluding any infectious or metabolic disorder that could be a cause of hepatic injury.

Table 2: Laboratory Values of 12 jaundiced Patients of the Second Category.

\begin{tabular}{|c|c|c|c|c|c|}
\hline \multirow{2}{*}{ Time After Starting } & \multirow{2}{*}{ Time After Stopping } & \multicolumn{3}{|c|}{$\nabla$} & \multirow{2}{*}{ Others } \\
\hline & & $\operatorname{ALT}(U / L)$ & $\operatorname{ALP}(U / L)$ & Bilirubin (mg/dL) & \\
\hline 0 & & 37 & 273 & 0.9 & $\begin{array}{l}\text { Analysis was taken as "P" therapy } \\
\text { started for OA }\end{array}$ \\
\hline 55 days & & & & & 'P' therapy was stopped \\
\hline 57 days & 0 & 694 & 575 & 5.9 & Itching, jaundice \\
\hline 60 days & 7 days & 270 & 736 & 4.5 & $\begin{array}{l}\text { Dark urine, pale stool, on the8th day } \\
\text { liver Biopsy was indicated }\end{array}$ \\
\hline 77 days & 14 days & 358 & 1688 & 3.3 & \\
\hline $3^{\text {th }}$ month & 20 days & 159 & 1240 & 2.1 & \\
\hline $4^{\text {th }}$ month & 1 month & 50 & 630 & 1.6 & \\
\hline $5^{\text {th }}$ months & 3 month & 61 & 291 & 0.7 & \\
\hline $6^{\text {th }}$ months & 4 months & 36 & 103 & 0.5 & \\
\hline \multicolumn{2}{|c|}{ Normal Values } & $<40$ & $<280$ & $<1.2$ & \\
\hline
\end{tabular}

N.B

凶: The symbol refers to mean average of laboratory values among 12 jaundiced patients of II category.

Alanine aminotransferase (ALT), alkaline phosphatase (ALP), Piroxicam ' $\mathrm{P}^{\prime}$

At the onset of symptoms, hepatic tests showed, elevated total amount of bilirubin, conjugated hyperbilirubinemia predominates unconjugated variant, normally, the total bilirubin level is less than $1.2 \mathrm{mg} / \mathrm{dL}$ (the reference range of direct bilirubin is $0.1-0.4 \mathrm{mg} /$ $\mathrm{dL}$ ) and approximately $70 \%$ is indirect (unconjugated) bilirubin. Conjugated hyperbilirubinemia $(>50 \%$ of the total bilirubin is direct) suggests hepatocellular dysfunction or cholestasis, Unconjugated hyperbilirubinemia $(>80 \%$ of the total bilirubin is indirect) suggests hemolysis or Gilbert syndrome, when the bilirubin level is above $25-30 \mathrm{mg} / \mathrm{d}$, extrahepatic cholestasis is an unlikely diagnosis; because the predominantly conjugated bilirubin is water soluble, it is easily excreted by the kidney in extrahepatic cholestasis. Levels of bilirubin in these patients begin gradually to be decreased after discontinuation of piroxicam therapy and normalization had occurred at the end of 4 th month (Table 2).

Regarding ALT and ALP levels, at the onset of symptoms, ALT serum is increased more than ALP, a week later serum ALT is decreased, but the serum of ALP is increased, then the end of the II week, levels of ALP are significantly elevated, compared to slightly increased ALT levels, and finally, the $20^{\text {th }}$ day of prioxicam withdrawal therapy reveals that levels of both enzymes begin gradually to be decreased and by the end of $4^{\text {th }}$ month are within normal limits in all patients, thus the $\mathrm{R}$ value which is employed to determine the relationship between ALT and ALP is 3.5, at the onset of symptoms, indicating a mixed hepatocellular-cholestatic pattern of injury, further elevations in ALP and a rapid decrease in 
ALT (by the $7^{\text {th }}$ day) yielding $R$ values of $<2$ ), generally when ALP is greater than twice the normal upper limit and $R \leq 2$, the type of injury is the cholestatic pattern, in hepatocellular pattern ALT is greater than twice the normal upper limit or $\mathrm{R} \geq 5$, and $2<\mathrm{R}<5$, it is the mixed type of injury (Table2). Based on the above data, onset of symptoms, negative serologic tests, biochemical analysis, $\mathrm{R}$ values, liver biopsy and taking considerations, that other causes of acute liver injury were effectively ruled out, as well as patients recovered steadily once therapy was stopped, complete recovery has occurred at the end of the 4 th month, all suggest that the likelihood that piroxiam at the dose of $20 \mathrm{mg} / \mathrm{d}$ for 2 month is the cause of the injury is highly probable. In fact, drug hepatotoxicity mechanisms could be classified into 2 classes:

a) Drugs that directly affect the liver, and usually is dose related, e.g., acetaminophen.

b) Idiosyncratic drug reactions: medications that promote hypersensitivity (immune) reactions due to either parent drug or its metabolite, the mechanism of piroxicam induced liver injury is not well known, but may be due to a toxic metabolic intermediate of piroxicam metabolism, which occurs largely in the liver.

\section{Conclusion}

a) Nocturnal acute pain of OA present in most (75\%) patients (above 55 years) of second group, and mainly dominant in women.

b) Piroxiacam, $10-20 \mathrm{mg} / \mathrm{d}$ for 2 month duration therapy is a good medicament for treatment of OA (II and III stage), with an ulcerogenic effect $(12,5 \%-25 \%)$ in the first and second categories respectively.

c) Piroxicam, $20 \mathrm{mg} / \mathrm{d}$ decreases the incidence of nocturnal pain and may be muscle spasticity, which suggests it as one of most effective drugs against pain at night.

d) Piroxicam, $20 \mathrm{mg} / \mathrm{d}$ is throughout to cause hepatic injury at the end of 8 th week of treatment in about $75 \%$ of patients belonging to the second category.

e) Piroxicam, $20 \mathrm{mg} / \mathrm{d}$ causes a mixed hepatocellularcholestatic pattern of injury, based on R values of ALT and ALP enzymes.

f) The hepatic injury induced by piroxicam is reversible as jaundiced patients completely recovered and laboratory values returned to their normal limits, by the end of $4^{\text {th }}$ month of piroxicam withdrawal therapy.

g) The mechanism of piroxicam-induced hepatotoxicity is unknown, but strongly believed to be idiosyncratic character, rather than dose related mechanism, piroxicam, $10 \mathrm{ma} / \mathrm{d}$ causes no hepatic injury.

h) It is recommended to control CBC indices as well as hepatic enzymes in patients on piroxicam therapy in the first 3-12 weeks in order to minimize its possible hepatotoxic effect.

\section{References}

1. Ahmad J, Odin JA (2017) Epidemiology and Genetic Risk Factors of Drug Hepatotoxicity. Clin Liver Dis 21 (1): 55-72.

2. Goodman ZD (2017) Phenotypes and Pathology of Drug-Induced Liver Disease. Clin Liver Dis 21(1): 89-101.

3. Gonzalez HC, Jafri SM, Gordon SC (2017) Management of Acute Hepatotoxicity Including Medical Agents and Liver Support Systems. Clin Liver Dis 21 (1): 163-180.

4. Benesic A, Gerbes AL (2015) Drug-induced liver injury and individual cell models. Dig Dis 33(4) : 486-491.

5. Fontana RJ, Hayashi PH, Gu J, Reddy KR, Barnhart H, et al. (2014) Idiosyncratic drug-induced liver injury is associated with substantial morbidity and mortality within 6 months from onset. Gastroenterology 147(1): 96-108.

6. Danan G, Benichou C (1993) Causality assessment of adverse reactions to drugs-I. A novel method based on the conclusions of international consensus meetings: Application to drug-induced liver injuries. J Clin Epidemiol 46(11): 1323-1330.

7. US Department of Health and Human Services Food and Drug Administration Center for Drug Evaluation and Research (CDER) Center for Biologics Evaluation and Research (CBER). Guidance for Industry, Drug-Induced Liver Injury: Premarketing Clinical Evaluation.

8. Lu TT, Makishima M, Repa JJ, Schoonjans K, Kerr TA, et al. (2014) Molecular Basis for Feedback Regulation of Bile Acid Synthesis by Nuclear Receptors. Mol Cell 6(3): 507-515.

9. Leise MD, Poterucha JJ, Talwalkar JA (2014) Drug-induced liver injury. Mayo Clin Proc 89(1): 95-106.

10. Lewis JH, Stine JG (2013) Nonsteroidal anti-inflammatory drugs and leukotriene receptor antagonists: pathology and clinical presentation of hepatotoxicity. In, Kaplowitz N, DeLeve LD, eds. Drug-induced liver disease. $3^{\text {rd }}$ Edition. Amsterdam: Elsevier, pp. 370-402.

11. Bhamidimarri KR, Schiff E (2013) Drug-induced cholestasis. Clin Liver Dis 17(4): 519-531.

12. Andrade RJ, Lucena MI, Kaplowitz N, García-Munoz B, Borraz Y, et al. (2006) Outcome of acute idiosyncratic drug-induced liver injury: Longterm follow-up in a hepatotoxicity registry. Hepatology 44(6): 15811588.

13. Lapeyre-Mestre M, Grolleau S, Montastruc JL (2013) Adsociation Française des Centres Régionaux de Pharmacovigilance (CRPV). Adverse drug reactions associated with the use of NSAIDs: a case/noncase analysis of spontaneous reports from the French pharmacovigilance database 2002-2006. Fundam Clin Pharmacol 27: 223-230.

14. Pandit A, Sachdeva T, Bafna P (2012) Drug induced hepatotoxicity: a review. J Appl Pharm Sci 2(5): 233-243.

15. Suzuki A, Brunt EM, Kleiner DE, Miquel R, Smyrk TC, et al. (2011)The use of liver biopsy evaluation in discrimination of idiopathic autoimmune hepatitis vs. drug-induced liver injury. Hepatology 54(3): 931-939.

16. Aithal GP (2011) Hepatotoxicity related to antirheumatic drugs. Nat Rev Rheumatol 7(3): 139-150.

17. Grossner T, Smyth EM, Fitzgerald GA (2011) Anti-inflammatory, antipyretic, and analgesic agents: pharmacotherapy of gout. In, Brunton LL, Chabner BA, Knollman BC (2011) Goodman \& Gilman's The pharmacological basis of therapeutics, $\left(12^{\text {th }}\right.$ edn). New York, USA, pp. 959-1004.

18. Fontana RJ, Seeff LB, Andrade RJ, Björnsson E, Day CP, et al. (2010) Standardization of nomenclature and causality assessment in druginduced liver injury: Summary of a clinical research workshop. Hepatology 52(2): 730-742. 
19. Bessone F (2010) Non-steroidal anti-inflammatory drugs: what is the actual risk of liver damage? World J Gastroenterol 16(45): 5651-5661.

20. Licata A, Calvaruso V, Cappello M, Craxì A, Almasio PL (2010) Clinical course and outcomes of drug-induced liver injury: nimesulide as the first implicated medication. Dig Liver Dis 42: 143-148.

21. Bjornsson E (2010) Review article: drug-induced liver injury in clinical practice. Aliment Pharmacol Ther 32(1): 3-13.

22. Reuben A, Koch DG, Lee WM (2010) Acute Liver Failure Study Group. Drug-induced acute liver failure: results of a U.S. multicenter, prospective study. Hepatology 52: 2065-2076.

23. Lammert C, Bjornsson E, Niklasson A, Chalasani N (2010) Oral medications with significant hepatic metabolism at higher risk for hepatic adverse events. Hepatology 51(2): 615-620.

24. Daly P, Donaldson PT, Bhatnagar (2009) HLA-B*5701 genotype is a major determinant of drug-induced liver injury due to flucloxacillin. Nat Genet 41(7): 816-819.

25. Wagner M, Zollner G, Trauner M (2009) New molecular insights into the mechanisms of cholestasis. J Hepatol 51(3): 565-580.

26. Food and Drug Administration F (2009) Guidance for Industry DrugInduced Liver Injury: Premarketing Clinical Evaluation. Center for Drug Evaluation and Research.

27. Chalasani N, Fontana RJ, Bonkovsky HL, Watkins PB, Davern T, et al (2008) Causes, clinical features, and outcomes from a prospective study of drug-induced liver injury in the United States. Gastroenterology 135: 1924-1934.

28. DA Working Group (2000) Drug-induced hepatotoxicity: white paper, post marketing considerations. PhRMA/FDA/AASLD: 1-29.

29. Tajiri K (2008) Practical guidelines for diagnosis and early mana-gement of drug-induced liver injury. World J Gastroenterol 14(44): 6774-6785.

30. Usui K, Oda Y, Kubota R, Negishi K, Uno K, et al. (2007) Clinical application of the leukocyte migration test and new diagnostic criteria for identifying causative agents in patients with drug-induced liver injury. Hepatogastroenterology 54(78): 1752-1757.

31. Rainsford KD (2007) Anti-inflammatory drugs in the $21^{\text {st }}$ century. Subcell Biochem 42: 3-27.

32. Hussaini SH, Farrington EA (2007) Idiosyncratic drug-induced liver injury: an overview. Expert Opin Drug Saf 6: 673-684.

33. Aithal GP, Day CP (2007) Nonsteroidal anti-inflammatory drug-induced hepatotoxicity. Clin Liver Dis 11(3): 563-575.

34. Lewis JH (2007) Nonsteroidal anti-inflammatory drugs and leukotriene receptor antagonists: pathology and clinical presentation of hepatotoxicity. In N Kaplowitz, LD DeLeve (Eds.) Drug-induced liver disease. New York, USA.
35. Vuppalanchi R, Liangpunsakul S, Chalasani N (2007) Etiology of newonset jaundice: How often is it caused by idiosyn-cratic drug-induced liver injury in the United States? Am J Gastroenterol 102(3): 558-562.

36. Pauli Magnus C, Meier PJ (2006) Hepatobiliary transporters and druginduced cholestasis. Hepatology 44(4): 778-787.

37. Polimeni G, Salvo F, Cutroneo P, Morreale I, Patrizio Caputi A (2006) Adverse reactions induced by NSAIDs and antibacterials: analysis of spontaneous reports from the Sicilian regional database. Drug Saf 29(5): 449-459.

38. Oude Elferink RPJ, Paulusma CC, Groen AK (2006) Hepatocanalicular transport defects: pathophysiologic mechanisms of rare diseases. Gastroenterology 130(3): 908-925.

39. Andrade RJ, Lucena MI, Fernández MC, Pelaez G, Pachkoria K, et al. (2005) Drug-induced liver injury: an analysis of 461 incidences submitted to the Spanish registry over a 10-year period. Gastroenterology 129(2): 512521.

40. Rostom A, Goldkind L, Laine L (2005) Nonsteroidal anti-inflammatory drugs and hepatic toxicity: a systematic review of randomized controlled trials in arthritis patients. Clin Gastroenterol Hepatol 3(5): 489-498.

41. Bertolami MC (2005) Mechanisms of hepatotoxicity. Arq Bras de Cardiol 85(5): 25-27.

42. Andrade RJ, Lucena MI AA (2004) HLA class II genotype influences the type of liver injury in drug-induced idiosyncratic liver disease. Hepatology 39(6): 1603-1612.

43. Rubenstein JH, Laine L (2004) Systematic review: the hepatotoxicity of non-steroidal anti-inflammatory drugs. Aliment Pharmacol Ther 20(4): 373-380.

44. Connor NO, Dargan PI, Jones AL (2003) Hepatocellular damage from non-steroidal anti-inflammatory drugs.QJM: International Journal of Medicine 96(11): 787-791.

45. Gutiérrez A, Enriquez R, Amorós F, Sillero C, Reyes A (2002) Acute hepatic and renal failure due to piroxicam use. Rev Esp Enferm Dig 94(3): 169-170.

46. Bessone F, Tanno H (2000) Hepatotoxicity induced by non-steroidal anti-inflammatory drugs Gastroenterol Hepatol 23: 200-205.

47. Poniachik J, Guerrero J, Calderón P, Smok G, Morales A, et al. (1998) Cholestatic hepatitis associated with piroxicam use. Case report. Rev Med Chil 126(5): 548-552.

48. Lee WM (1995) Drug-induced hepatotoxicity. N Engl J Med 333(17): 1118-1127.

49. Paterson D, Kerlin P, Walker N, Lynch S, Strong R (1992) Piroxicam induced submassive necrosis of the liver. Gut 33(10): 1436-1438.

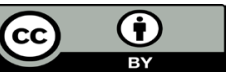

This work is licensed under Creative Commons Attribution 4.0 License

Submission Link: http://biomedres.us/submit-manuscript.php

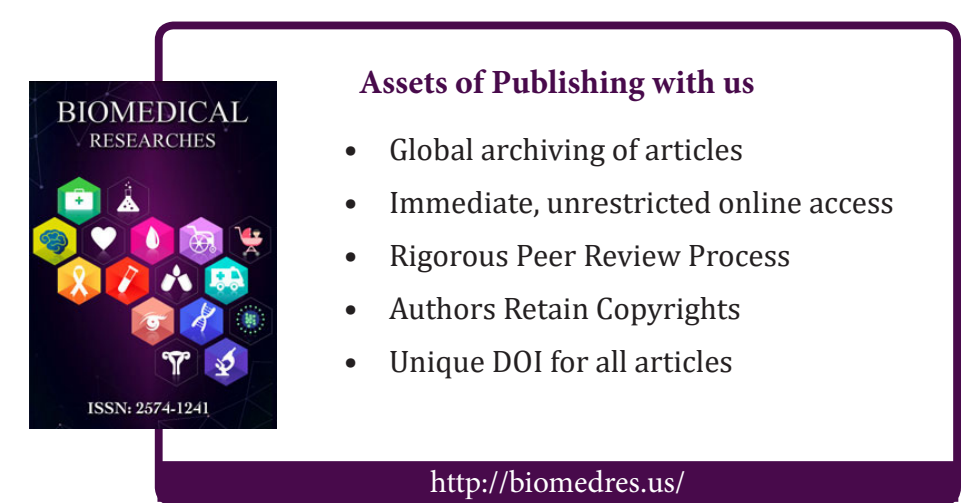

